\title{
How do the questions asked affect suspects' perceptions of the interviewer's prior knowledge?
}

\author{
Meghana Srivatsav ${ }^{1,2}$ (c) | Timothy J. Luke \\ Pär Anders Granhag ${ }^{1}$ | Aldert Vrij ${ }^{2}$ \\ ${ }^{1}$ Department of Psychology, University of Gothenburg, Gothenburg, Sweden \\ ${ }^{2}$ University of Portsmouth, Portsmouth, UK
}

\section{Correspondence}

Meghana Srivatsav, Department of

Psychology, University of Gothenburg. Box

500, Gothenburg 405 30, Sweden.

Email: meghana.srivatsav@psy.gu.se

Funding information

Erasmus Mundus Joint Doctorate Programme: The House of Legal Psychology (EMJD-LP), Grant/Award Numbers: 2016-1339, 2013-0036

\begin{abstract}
The aim of this study was to understand if guilty suspects' perceptions regarding the prior information or evidence held by the interviewer against the suspect could be influenced through the content of the investigative questions. To test this idea, we explored three question-phrasing factors that we labeled as topic discussion (if a specific crime-related topic was discussed or not), specificity (different levels of crime-related details included in the questions), and stressor (emphasis on the importance of the specific crime-related detail in the questions). The three factors were chosen based on relevance theory, a psycholinguistic theory that explores how people draw inferences from the communicated content. Participants $(N=370)$ assumed the role of the suspect and read a crime narrative and an interview transcript based on the suspect's activities. After reading the narrative and the transcripts, participants responded to scales that measured their perception of interviewer's prior knowledge (perceived interviewer knowledge [PIK]) regarding the suspects' role in the crime, based on the questions posed by the interviewer in the transcripts. Of the three factors tested, we found that questioning about a specific crime-related topic (topic discussion) increased their PIK.
\end{abstract}


This study is the first to explore the underlying mechanisms of how suspects draw inferences regarding the interviewer's prior knowledge through the content of the investigative questions adopting concepts of psycholinguistic theory.

\section{KEYWORDS}

interviewing guilty suspects, investigative questions, police interviewing, relevance theory, suspects' inferences

\section{INFLUENCE OF QUESTION CONTENT IN POLICE INTERVIEWS ON SUSPECTS' INFERENCES REGARDING INTERVIEWER'S PRIOR KNOWLEDGE}

Imagine that you overhear the following conversation between two friends:

John: Was Lisa at Rob's party last night?

Mike: The spouses weren't invited.

From just this short exchange, you probably learned a great deal about what is going on. You can probably tell that (a) Rob threw a party last night; (b) John knew that Mike was either present at Rob's party or had information regarding the party; (c) John was not at the party; (d) Lisa is the spouse of someone present at the party; (e) John and Mike both know who Lisa is; (f) Mike knows that John knows who Lisa is; (g) Lisa was not at the party; and (h) John did know whether Lisa was at the party prior to hearing Mike's response, but he presumably now knows she was not.

None of these enumerated conclusions are likely to surprise you, but you may have drawn these inferences so easily and spontaneously that you hardly noticed how much you learned from just two utterances. Strikingly, it is easy to arrive confidently at these inferences despite their not being directly stated. Superficially, Mike's response does not actually answer John's question, and yet it is not difficult to understand the implication and see how he has, in fact, provided an adequate answer to John's inquiry. With a little reflection on personal experience, it is easy to see that human communication ubiquitously relies on this kind of "reading between the lines" in order to infer unstated assumptions. We draw inferences and make implications in this manner virtually every time we communicate with other people-cutting logical corners and leaving many assumptions unsaid, with little or no loss of understanding.

How exactly do we do this? Psycholinguists have endeavored, for several decades, to provide an answer to this question (Grice, 1975; Sperber \& Wilson, 1995). This area of psycholinguistics-the study of pragmatic implicature-is concerned with human communication in general, but here, we are particularly interested in a specific context in which inferred and implied information can be of especially high practical value: police interviews and interrogations.

We are not the first to argue that pragmatic implication and inference have important consequences in police interviewing. For example, Kassin and McNall (1991) have argued that downplaying the moral seriousness of a crime or providing reasonable explanations for why the suspect may have committed the offense-a common accusatory interrogation technique known as minimization (for a description of this technique, see Kassin et al., 2010)-can pragmatically imply that the suspect will receive more lenient treatment if he or she confesses to the crime in question, even though this promise is never directly offered. More recently, Brimbal, Hartwig, and Crossman (2017) have reviewed existing literature on the theoretical aspects of how question content could influence suspects' perceptions regarding the evidence or prior crime-related information held by the interviewer against them through pragmatic inferences. However, to the best of our knowledge, there has been no previous empirical research examining how 
suspects might use the content and phrasing of interview questions to draw inferences about what the interrogator knows and what evidence he or she might possess-that is, what we might call perceived interviewer knowledge (PIK).

Perceived interviewer knowledge is theorized to be an important determinant of a suspect's behavior in police interviews (Granhag \& Hartwig, 2015). Specifically, PIK may influence a suspect's perception of how best to act in order to convince the interviewer that he or she is innocent. Consequently, the suspect's strategy for convincing the interviewer impacts the amount of potentially incriminating information the suspect is prone to disclose (Granhag, Hartwig, Mac Giolla \& Clemens, 2015; Granhag, Rangmar, \& Strömwall, 2015; Oleszkiewicz, Granhag, \& Kleinman, 2014). Currently the research points out to how suspects draw inferences regarding the interviewer's prior knowledge primarily as a function of evidence disclosure-that is, the interviewer directly stating the evidential basis of his or her knowledge (see Granhag \& Hartwig, 2015; Granhag, Hartwig, et.al, 2015; Granhag et al., 2015). However, given how readily people draw inferences about others' knowledge and motives from what they say and how they say it, it is plausible that suspects draw inferences about the interviewer's knowledge from the mere content and phrasing of interview questions-without any evidence being disclosed.

We propose that suspects, particularly guilty suspects use the content and phrasing of interview questions to draw inferences about the interviewer's crime relevant knowledge (viz. how much they know about what actually happened during a crime) and the interviewer's objectives. More specifically, we hypothesize that (a) the mere act of asking questions about a given topic will suggest the interviewer is more knowledgeable about that topic; (b) when interview questions request more specific information, people will infer that the interviewer is more knowledgeable; and (c) when the interviewer emphasizes the importance of requested information, people will infer that the interviewer is less knowledgeable. We derive these broad predictions from past psycholinguistic literature, which we review below.

\section{I PRAGMATIC INFERENCES FROM QUESTIONS: RELEVANCE THEORY}

Building on Grice's (1975) influential work on the "logic of conversation," Sperber and Wilson (1995) proposed relevance theory to better understand how people interpret and draw pragmatic inferences from utterances. The theory places special emphasis on the relevance of information, as its name suggests. Grice (1975) postulated that conversations operate according to, among several implicit rules, a principle that prescribes that each utterance is relevant. However, Grice was not specific about what was meant by "relevant." Relevance theory aims to clarify the construct of relevance and explicate its role in pragmatic inference and implication.

According to relevance theory, we draw inferences with the assumption that speakers are trying to make understanding their utterances as easy as possible. That is, we implicitly assume that the simplest, most easily available but most informative interpretation of the utterance is the correct one. This does not mean that the intended meaning of any given utterance is simplistic; rather, it means that we assume utterances are informative and then guess about what also must be true in order for the utterance to be informative in whatever context in which we are operating. In the example above, Mike's response ("The spouses weren't invited.") does not contain a literal answer to John's question. However, we can obtain the intended meaning by assuming that Mike's utterance does in fact answer the question and deducing what else could be true in order to make the utterance informative. Relevance theory posits that we implicitly work backwards from the assumption that the utterance does contain an answer and therefore (correctly) hypothesize that Lisa must be a spouse of someone at the party, as that is the most straightforward explanation as to how the superficially uninformative utterance actually contains relevant information (for a more thorough treatment of relevance theory, see Sperber \& Wilson, 1995).

When John asks the question to Mike, the relevance of his question not only depends on the context but also for whom the answer to the question is relevant. In this case, Mike can assume that the answer to John's question is relevant to John and respond accordingly. When John asks Mike if Lisa was present at Rob's party the previous night, the question already provides Mike the cues to assume what would be relevant to John as a response. In other words, John has already indicated to Mike that he holds some information about the context (i.e., Rob's party the 
previous night) without explicitly stating what he knows, and the information he specifically seeks (i.e., Lisa's presence at the party). Mike now has information that a response informing John if Lisa was present at the party or not would be relevant to John. Hence relevance theory posits that we make inferences regarding what the speaker already knows and what they intend to learn, using the content of questions and context. We then decide an appropriate response based on these inferences and assumptions (for a more complete description of interrogatives in relevance theory, see, Clark, 1991).

\section{2 | PRAGMATIC INFERENCES DURING POLICE INTERVIEWS}

Drawing from our understanding of how people draw inferences in an everyday context as illustrated in the examples above, we will now apply this understanding to inferences drawn by guilty suspects in police interviews. Specifically, we will examine factors related to the content of questions which may influence guilty suspects' inferences regarding how much prior knowledge the interviewer already holds regarding the suspect's role in crime namely: (a) topic discussion-discussing about a specific crime-related topic, (b) level of specificity-including specific crimerelated details, and (c) stressor-emphasizing on specific crime-related detail. From our understanding of relevance theory, we tested these three factors particularly because they had a strong theoretical foundation, and they seemed to be plausible factors that could influence people's inferences.

Earlier, we introduced John and Mike as friends having a conversation in an everyday scenario. We will now discuss the factors mentioned above with the following context where John is a detective and Mike is a suspect in the case of Lisa's disappearance from Rob's party:

John: What was Lisa doing while you were at Rob's party last night?

Mike: I think she was at the movies with a friend.

\subsection{Topic discussion}

When a specific topic is merely discussed or questioned about, this indicates that the speaker discussing or asking the question about that topic has some amount of background knowledge about the topic (Clark, 1991). For example, in the exchange above, Mike being the suspect could infer from John's question that John has some knowledge about the context (Rob's party) and the people involved, but he does not seem to know what Lisa was specifically doing and is hence seeking new information. Here, the fact that John questioned about the topic of Rob's party suggests that John had some prior information about Rob's party, although the amount of prior information held by John is open for interpretation. That is, in this case, Mike could infer that John could have more information than he is letting on or that he has only the basic information that Mike was present at Rob's party that took place the previous night.

\section{2 | Level of specificity}

The information that is relevant to John in this context is different and John is now looking for information regarding Lisa's activities and not her whereabouts as in the earlier context. Thus, if a question is more general, such as a request for information (Sperber \& Wilson, 1995), like John's question to Mike above, the assumption is that the speaker has relatively little information about the background (Jacobssen, 2010) and a response with basic information would suffice as a relevant response. That is, Mike could infer that John is asking a question about Lisa's activities because he does not have any information regarding her activities, indicating low prior knowledge. 
However, imagine John instead asked a more specific question such as, "Was Lisa at the movies when you were at Rob's party last night?" Here, it seems that John may hold some specific information about Lisa's whereabouts and is seeking confirmation. The idea is that if John had asked a broader question about Lisa's whereabouts (Where was Lisa last night?), it seems like John has low prior information regarding her activities. However, if John asked Mike a question with some specific detail regarding her activities, it could indicate that John could probably be asking for a confirmation about the specific detail (that Lisa was at the movies), and that John already has this information with him. A relevant response in this context would be Mike confirming or denying Lisa being at the movies or possibly providing specific information about her location. A response addressing the detail in the question with more specific details would be considered as a relevant response (Sperber \& Wilson, 1995).

It is also possible for a speaker to communicate incorrect information through a question. When the speaker asks a specific question with correct details like in the example illustrated above, the inference is more straightforward. However, if the specific question contains incorrect details, it could lead to an ambiguous situation where the listener can make different inferences regarding why the speaker has communicated incorrect information. For example, the listener can infer that the speaker is ignorant about the correct information in the context and hence requires a response with the correct detail to be relevant; or that the speaker holds some prior information for which they require a response that might confirm or decline their prior knowledge as correct. For instance, if John were to ask Mike "Was Lisa at the bar when you were at Rob's party last night?" Mike could infer either that John may be ignorant of the correct information (that Lisa was at the movies) or may strategically be trying to gather correct information from Mike through his response. Mike could respond by telling John that Lisa was at the movies and not at a bar. He might even perceive that John wants Mike to give him correct details as a confirmation on the information he already holds. Hence, a relevant response when the listener is presented with incorrect details in a question would be providing the correct detail to either offer the speaker with new information or confirm already known information (Sperber \& Wilson, 1995). Because incorrect information could plausibly result in qualitatively different inferences, below, we make competing predictions in our study, which we refer to as the Ignorance hypothesis and Strategic Inference hypothesis.

\section{\begin{tabular}{l|l}
2.3 & Stressor
\end{tabular}}

Now let us consider how emphasis on specific details within questions could influence inferences. If John were to emphasize the importance of a specific detail (e.g., Lisa being at the movies, or the bar) in any of the questions above, Mike would infer that a response regarding the detail would be most relevant for John and that John is seeking information related to that specific detail since he is bringing Mike's attention to that detail. For instance if John were to ask, "Mike, this information is critical: Was Lisa at the movies when you were at Rob's party last night?" Mike could infer that John is seeking information specifically about Lisa being at the movies due to the emphasis John placed on this information. So a response confirming or denying this particular detail would be the most relevant to John. Emphasizing particular content in a question would lead to the inference that the speaker holds some information regarding that detail and probably less or no information about any other aspect of the context.

\section{3 | THE PRESENT STUDY}

Based on the theoretical understanding described above, we designed the present study to examine factors that may influence inferences people tend to draw regarding crime-related knowledge of the interviewer from what they say. As illustrated in the examples, we tested how changing small details within a question would influence what the suspect thinks the interviewer already knows. We specifically tested three question content factors that could influence the inferences suspects made regarding the interviewer's prior knowledge namely topic discussion, specificity and stressor (described later). 
As discussed early on, we predicted that questioning about a topic would lead the suspect to infer a higher knowledge about that topic. When the interviewer asked questions with specific detail we predicted that the correct details would infer more knowledge about that detail and the incorrect details may infer less knowledge. It may be asked corroboratory purposes about that detail as well. We also predicted that emphasizing specific detail in the questions leads to an inference of knowledge only about the emphasized detail and hence a lower knowledge about the overall crime. We labeled our predictions as knowledge inference and goal inference hypotheses based on the specific measures that tested these hypotheses.

The procedure and hypotheses were preregistered on the Open Science Framework (OSF; osf.io/7z6sf) prior to data collection and the analysis plan was preregistered prior to analysis.

\section{4 | METHOD}

\subsection{Independent variables}

The three main factors we tested in this study are as follows:

Topic discussion: whether or not the interviewer addressed the specific crime-related activity and asked questions about the topic.

Level of specificity: the amount of crime-related detail that was included in the question. Three levels of specificity were tested. (a) General-consisted of broad questions without specific crime-related details. (b) Specific correctconsisted of questions with specific correct crime-related details, and (c) Specific incorrect-consisted of questions with specific incorrect crime-related details.

Stressor: emphasis on specific detail that the interviewer placed on in the question.

\section{2 | Design}

The study used a 3 (question type: general vs. specific correct vs. specific incorrect-between subjects) $\times 2$ (stressor conditions: without stressor vs. with stressor-between subjects) $\times 2$ (topic discussion: discussed vs. not discussedwithin subjects) mixed model design.

\section{3 | Participants}

We recruited $N=434$ MTurkers as participants, to account for exclusions. We excluded the data of 64 participants who failed an instructional manipulation check (IMC) and attention check questions included in the survey. For the IMC, participants had to read an irrelevant paragraph about a certain topic that ended with an instruction to ignore the content they read and respond with a plus sign. For the attention checks, participants answered simple factual questions regarding the content of the transcript they had read. We excluded data from participants who failed to respond correctly to two or more of these three attention questions. Our final sample included $N=370$ (307 females and 63 males; $19-72$ years; $M=37.89, S D=11.51$ ) participants. Power calculations indicated that a sample size of this size was sufficient to detect an effect of $f=0.21$ with $95 \%$ power.

\section{4 | Procedure}

The study was administered online using Qualtrics. Participants completed a form consisting of basic demographic details such as their gender, age, education, and ethnicity. A brief description of the study and instructions to take part in the study followed this. 
Participants read a crime narrative with the background story that placed them in the scenario of the crime. The crime narrative was about an illegal guns trading where the suspect who poses as a mechanic carries out illegal activities to transport gun shipments. They are encouraged to immerse themselves into the role of the suspect. The narrative consists of three activities the suspect carries out as part of the crime: (a) shipment of the illegal gun parts in an art museum through a truck driver; (b) obtaining illegal paperwork for shipments from a Police Officer working from the inside; and (c) shipment of the illegal gun parts on a shipping dockyard with the help of the loading manager. The activities were written such that they could be carried out in any order. We designed the activities in this way so that we could randomize the order of presentation to reduce order effects. Participants responded to attention check questions between these scripts.

After they completed reading the crime narrative, they read interview transcripts regarding the suspect's activities and the transcripts represented the exchange between the suspect and the detective. They responded to a rating scale about how much prior knowledge they thought the interviewer had on a scale of 0 (low) to 10 (high) after they read the transcript regarding each crime-related activity. We referred to this as the Knowledge Inference Scale. The rating scale was presented as a set of three questions; one for each specific activity. The scale consisted of how much prior knowledge they thought the interviewer had about the activity that was questioned about in the transcript presented and also about the other two activities that were either presented earlier or not presented. Participants responded to this scale three times in total (once after each topic interview) adding up to nine measures of PIK. We coded the measures as topic discussion (whether the specific interview transcript for the specific activity was discussed or not discussed).

Once they completed reading and responding to all the interview transcripts and the rating scales, they were presented with another rating scale measuring to what extent the suspect believed the interviewer wanted to obtain new information, corroborate existing information, and obtain a confession. We referred to this as the Goal Inference Scale. The scale was rated between 0 indicating Not Likely at all and 10 indicating Highly Likely. After they responded to this scale the study was complete. They are debriefed about the purpose of the study following which they receive their compensation for participating in the study.

\section{5 | Hypotheses}

\subsection{1 | Knowledge inference hypotheses}

These predictions are based on the extent to which the interviewer's questions imply previous knowledge about each topic; we made the following predictions for the suspect's PIK.

1. PIK measured by the three knowledge inference scales in the general no-stressor condition will be low on the theme for which the interview has been presented and low on the themes for which the interview has not been presented yet. That is, we predicted a main effect of topic discussion in the general, no stressor condition.

2. PIK in the general stressor condition will be moderate on the theme for which the interview has been presented and low on the themes for which the interview has not been presented yet. That is, we predicted an interaction between topic discussion and stressor such that stressors would increase PIK when the topic had been addressed.

3. PIK in the specific correct no stressor condition will be moderate on the theme for which the interview has been presented and low on the themes for which the interview has not been presented yet. That is, we predicted an interaction between specific correct details and topic discussion such that specific correct details would increase PIK when the topic had been addressed.

4. PIK in the specific correct stressor condition will be high on the theme for which the interview has been presented and moderate on the themes for which the interview has not been presented yet. Here, we predict an 
interaction between specific correct details, stressor and topic discussion such specific correct details with a stressor will increase PIK when the topic had been addressed.

As discussed in the earlier sections where the detective asks questions with incorrect information, this represents a more complex inferential situation leading to competing hypotheses. We hence have competing hypotheses, which we refer to as ignorance hypothesis and strategic inference hypothesis.

5. Ignorance hypothesis: (a) PIK in the specific incorrect, no stressor condition will be low on the theme for which the interview has been presented and low on the themes for which the interview has not been presented yet. That is, we did not predict an effect of topic discussion in the specific incorrect, no stressor condition. (b) PIK in the specific incorrect stressor condition will be low on the theme for which the interview has been presented and low on the themes for which the interview has not been presented yet. That is, the ignorance hypothesis predicts a negative effect of topic discussion in the specific incorrect, stressor condition.

6. Strategic inference hypothesis: (a) PIK in the specific incorrect no stressor condition will be moderate on the theme for which the interview has been presented and moderate on the themes for which the interview has not been presented yet. That is, the strategic inference hypothesis predicted a positive effect of topic discussion in the specific incorrect, no stressor condition. (b) PIK in the specific incorrect stressor condition will be high on the theme for which the interview has been presented and moderate on the themes for which the interview has not been presented yet. That is, the strategic inference hypothesis predicted a positive effect of topic discussion in the specific incorrect, stressor condition and an interaction between these three factors, such that stressors would increase PIK.

\subsection{2 | Goal inference hypotheses}

These predictions are based on the extent to which the interviewer's questions imply specific motivations to gather new information, corroborate with existing information, or obtain a confession. We made the following predictions:

7. Perception of interviewer's motivation for new information will be higher in general and specific incorrect conditions in comparison to the specific correct condition.

8. Perception of interviewer's motivation for admissions/confessions will be higher in the specific correct condition in comparison to general and specific incorrect conditions.

9. Perception of interviewer's motivation for corroborating information will be higher in general conditions in comparison to specific correct and specific incorrect conditions.

\section{5 | RESULTS}

\subsection{Hypotheses tests}

\subsection{1 | Knowledge inference hypotheses}

To test the knowledge inference hypotheses, we fit a linear mixed effects model with specificity, stressor, topic discussion, and their interaction terms as fixed effects. We also included random intercepts for subjects nested under different conditions, order in which the topics/crime phases were discussed, and for each of the three crime phases.

As predicted, we found a significant main effect of topic discussion on PIK such that guilty suspects inferred that the interviewer had more knowledge about a crime-related topic that was questioned about that seemed to increase 


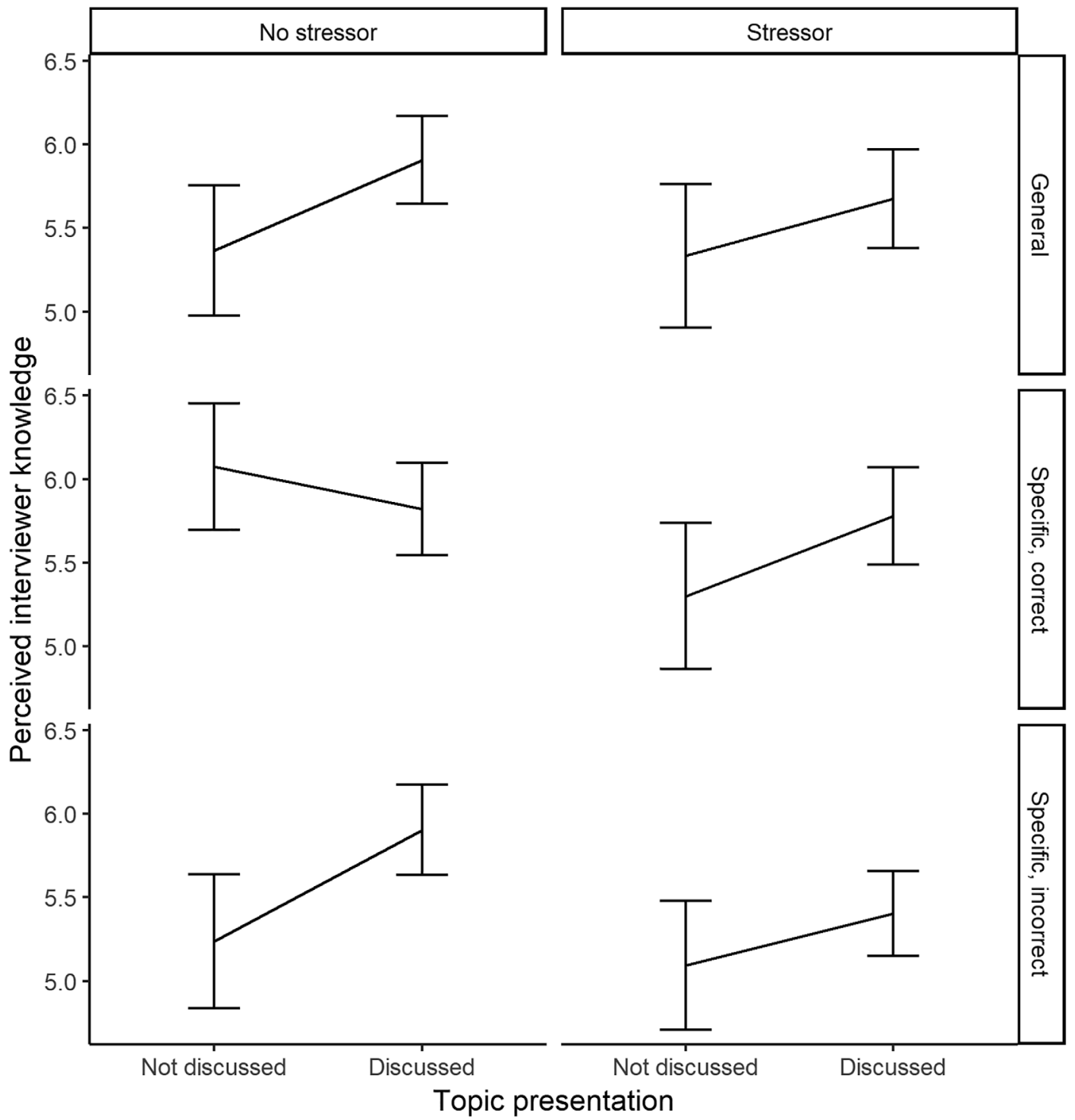

FIGURE 1 Means of PIK across topic discussion, specificity, and stressor conditions. Note: Each panel represents an effect of topic discussion, that is, whether a crime-related topic is discussed or not across the specificity and stressor conditions. The bars represent the increase in mean values across the specificity and stressor conditions as a function of topic discussion. PIK, perceived interviewer knowledge

PIK of other topics that were not questioned about. We did not observe the predicted effects for level of specificity and stressors. However, we noted that emphasizing on the questions content with stressors seemed to overall reduce the suspect's perception of the interviewer's background knowledge in all the specificity conditions, though this pattern was not significant. We also observed that specific correct details seemed to influence PIK in the expected direction such that the suspect inferred slightly higher interviewer knowledge in this condition in comparison to general and specific incorrect details (see Figure 1 and Tables 1 and 2).

A three-way interaction was observed between topic discussion, stressor, and specific correct conditions (see Table 1). As can be seen in Figure 1, this interaction appears to be driven by mean for unaddressed topics in the 
TAB LE 1 Mixed effects model: Perception of interviewer's knowledge as a function of level of specificity, stressor, and topic discussion

\begin{tabular}{|c|c|c|c|c|c|}
\hline \multicolumn{6}{|l|}{ Fixed effects } \\
\hline Term & $b$ & $S E$ & $d f$ & $t$ & $p$ \\
\hline Intercept (general) & 5.37 & 0.54 & 3.4 & 9.99 & $<.001$ \\
\hline Specific correct & 0.71 & 0.36 & 592.9 & 1.95 & .051 \\
\hline Specific incorrect & -0.08 & 0.37 & 592.7 & -0.2 & .83 \\
\hline With stressor & -0.06 & 0.37 & 592.7 & -0.16 & .86 \\
\hline Topic discussion & 0.51 & 0.18 & $2,952.1$ & 2.78 & $<.001$ \\
\hline Specific correct: with stressor & -0.68 & 0.52 & 588.7 & -1.3 & .19 \\
\hline Specific incorrect: with stressor & -0.17 & 0.53 & 592.4 & -0.32 & .74 \\
\hline Specific correct: topic discussed & -0.76 & 0.26 & $2,952.1$ & -2.97 & $<.001$ \\
\hline Specific incorrect: topic discussed & 0.08 & 0.26 & $2,952.1$ & 0.32 & .74 \\
\hline With stressor: topic discussed & -0.15 & 0.26 & 2,952 & -0.57 & .56 \\
\hline Specific correct: with stressor: topic discussed & 0.83 & 0.37 & 2,952 & 2.28 & $<.01$ \\
\hline Specific incorrect: with stressor: topic discussed & -0.07 & 0.37 & 2,952 & -0.19 & .85 \\
\hline \multicolumn{6}{|l|}{ Random effects } \\
\hline Term & $S D$ & & & & \\
\hline Subjects & 1.68 & & & & \\
\hline Order of topics discussed & 0.14 & & & & \\
\hline Topics discussed & 0.81 & & & & \\
\hline
\end{tabular}

Note: $t$-tests used Satterthwaite approximated degrees of freedom. Both factors use treatment contrasts. The reference group for topic discussion is topic discussed, specificity condition is general and stressor is with stressor.

TABLE 2 Descriptive statistics for perception of interviewer knowledge

\begin{tabular}{|lllllll|}
\hline Specificity & Topic discussion & Stressor & Mean & SD & SE & Observations \\
\hline General & Not discussed & Not present & 5.36 & 2.76 & 0.20 & 192 \\
\hline General & Not discussed & Present & 5.33 & 2.99 & 0.22 & 186 \\
\hline General & Discussed & Not present & 5.91 & 2.63 & 0.13 & 384 \\
\hline General & Discussed & Present & 5.67 & 2.89 & 0.15 & 372 \\
\hline Specific correct & Not discussed & Not present & 6.07 & 2.67 & 0.19 & 192 \\
\hline Specific correct & Not discussed & Present & 5.30 & 3.01 & 0.22 & 183 \\
\hline Specific correct & Discussed & Not present & 5.82 & 2.76 & 0.14 & 384 \\
\hline Specific correct & Discussed & Present & 5.78 & 2.84 & 0.15 & 366 \\
\hline Specific incorrect & Not discussed & Not present & 5.24 & 2.72 & 0.20 & 177 \\
\hline Specific incorrect & Not discussed & Present & 5.09 & 2.64 & 0.20 & 180 \\
\hline Specific incorrect & Discussed & Not present & 5.90 & 2.59 & 0.14 & 354 \\
\hline Specific incorrect & Discussed & Present & 5.40 & 2.46 & 0.13 & 360 \\
\hline
\end{tabular}

Note: Higher values indicate higher perceived interviewer knowledge (PIK).

specific correct, no stressor condition. This cell stands out from the general pattern of results (i.e., that topic discussion tends to increase PIK). Because of its unusualness and deviation from the general pattern, we suspect this effect is spurious and may be due to sampling error. 
TABLE 3 Main effects and interactions of factors on goal inferences

\begin{tabular}{|llllll|}
\hline Goal inference & Factors & Sum of squares & df & $\boldsymbol{F}$ & $\boldsymbol{p}$ \\
\hline New information & Specificity & 34.02 & 2,364 & 2.58 & .07 \\
& Stressor & 0.44 & 1,364 & 0.06 & .79 \\
\hline \multirow{2}{*}{ Confession } & Specificity $\times$ stressor & 7.14 & 2,364 & 0.54 & .58 \\
\hline Corroboration & Specificity & 0.612 & 2,364 & 0.04 & .96 \\
\hline & Stressor & 14.229 & 1,364 & 1.67 & .19 \\
\hline & Specificity $\times$ stressor & 3.909 & 2,364 & 0.23 & .79 \\
\hline & Specificity & 0.884 & 2,364 & 0.13 & .88 \\
\hline & Stressor & 0.076 & 1,364 & 0.02 & .88 \\
\hline
\end{tabular}

\section{2 | Goal inference hypotheses}

We ran two-way ANOVAs to assess the effect of specificity and stressors on the goal inference measures. We predicted a main effect of the specificity conditions on the goal inference measures. We did not predict a main effect of stressor or an interaction between levels of specificity and stressor and included the interaction terms for exploratory purposes. The analyses show that there were no significant main effects or interactions (see Table 3). The hypotheses were not supported.

\section{6 | DISCUSSION}

The present study assessed how different content in investigative questions influence guilty suspects' perception of interviewer's prior knowledge. Specifically, we tested how questioning about a specific topic, including varying amount of detail and emphasizing on particular detail in a question would influence suspects' perceived interviewer's knowledge. We found that questioning about a specific topic, what we referred to as topic discussion, led the suspect to perceive the interviewer to have higher knowledge about that topic in comparison to other topics. However, we did not find support for our predictions about specific crime-related details and emphasis on the content in the questions.

Altering specific crime-related details within questions did not seem impact PIK the way we expected. In trying to explain this outcome, we posit that questioning about a specific topic is driving the effect to a larger degree and limiting the effects of specific content within the question. That is, guilty suspects could be making inferences regarding what the interviewer might know about their crime-related activities primarily based on the fact that a topic has been discussed, rather than on the specific details mentioned by the interviewer.

Although the effect size was small (equivalent to $d=0.12$ ), this finding could indicate that questioning about a topic irrespective of the amount of crime-related detail held by the interviewer could indicate some prior knowledge in comparison to not talking about the topic at all. Previous research indicates that guilty suspects infer higher prior interviewer knowledge and reveal information when they are confronted with evidence but infer less or no prior knowledge and withhold information when not confronted with evidence information (Tekin, Granhag, Strömwall, Vrij, \& Hartwig, 2015; Tekin, Granhag, Strömwall, Vrij, 2016; Luke, Dawson, Hartwig, \& Granhag, 2014). The findings from this study show us that suspects might infer some amount of prior knowledge about an activity irrespective of the crime-related detail they are presented with in the questions. That is, there is some difference between suspects' inferences when a specific activity is addressed with or without presenting incriminating details than when the activity is not addressed.

Delving into our understanding of inferences from questions with the concepts of relevance theory, we can suggest that question content possibly influences shifts in what becomes relevant from the information revealed by the 
interviewer in the questions. This means that for a guilty suspect, talking about a certain crime-related activity that they have committed seems to shift their focus to the overall context of the activity rather than the specific details about the activity. It is possible that guilty suspects draw inferences regarding what information the interviewer holds based on the overall context rather than the details in the questions.

\subsection{Limitations and future directions}

There are at least three major limitations to the present study. First, the setup of the study could have reduced the motivation of the participants that could be higher in real-world scenarios. Actively interviewing a suspect would probably lead to interesting findings adding to the findings from this study. However, since we were only trying to understand how people draw inferences from linguistic changes, regardless of motivation, this limitation is not fatal to our conclusions.

Second, it is possible that the manipulations of the specific details in the questions may not have been strong enough to elicit the outcome predicted. For instance, the general and specific conditions had questions related to each specific activity with only a small amount of details changed between the conditions. It is possible that this could have resulted in the very small differences between the conditions. If we probably broaden the scope of the question in the general condition and narrowed down further towards the actual activity in the specific condition, it could result in the outcome that we predicted. It is hence important to investigate if constructing questions with more obvious content differences would influence suspects' inferences.

Third, we limited the factors we tested to crime-related content in questions and did not explore other surrounding determinants, for example, the tone of the interviewer. For example, if the interviewer used a more accusatory tone during questioning versus a more neutral tone (Srivatsav, Luke, Granhag, \& Vrij, 2019). Exploring such factors surrounding an interview could provide a broader understanding of how an interviewer can influence a suspect's inferences regarding their prior knowledge.

Finally, we wish to comment on the failure to support the goal inference hypotheses. We speculate that the goal inference hypotheses were not supported for two reasons. The first reason is that we included one question to test each goal inference making it a relatively weak measure of the construct. The second reason is the fact that participants only imagined themselves in the role of the suspect and read transcripts, so it was probably not possible for them to actively infer what the goal of the interviewer might be, since they were not directly questioned.

\section{$6.2 \mid$ Conclusions}

The present study provides an initial investigation of how the content of interview questions can potentially influence the inferences of suspects in police interviews. With this study, we have adopted a psycholinguistic perspective to understand how interviewers can influence guilty suspects' inferences regarding the information held by the interviewer. Guilty suspects seem to assess the content of questions to draw inferences regarding the interviewer's prior knowledge actively during the interview irrespective of the amount of information revealed during the interview. While existing psycho-legal literature have explored how guilty suspects' PIK could be influenced as a function of disclosing evidence, this study has tried to test if basic content in the question could influence their inferences. This study can provide researchers novel predictions about how suspects actively assess information during an interview.

\section{ACKNOWLEDGMENTS}

This research is supported by a fellowship awarded from the Erasmus Mundus Joint Doctorate Programme: The House of Legal Psychology (EMJD-LP) with Framework Partnership Agreement (FPA) 2013-0036 and Specific Grant Agreement (SGA) 2016-1339 to Meghana Srivatsav. 


\section{ORCID}

Meghana Srivatsav (1D) https://orcid.org/0000-0001-7490-4916

Aldert Vrij (iD) https://orcid.org/0000-0001-8647-7763

\section{REFERENCES}

Brimbal, L., Hartwig, M., \& Crossman, A. M. (2017). The effect of questions on suspects' perception of evidence in investigative interviews: What can we infer from the basic literature? Polygraph \& Forensic Credibility Assessment, 46, 10-39.

Clark, W. (1991). Relevance theory and the semantics of non-declarative sentences. (PhD thesis). University College London.

Granhag, P. A., \& Hartwig, M. (2015). The strategic use of evidence (SUE) technique: A conceptual overview. In P. A. Granhag, A. Vrij, \& B. Verschuere (Eds.), Deception detection: Current challenges and new approaches (pp. 231-251). New York, NY: John Wiley \& Sons.

Granhag, P. A., Hartwig, M., Mac Giolla, E., \& Clemens, F. (2015). Suspects' verbal counter-interrogation strategies: Towards an integrative model. In P. A. Granhag, A. Vrij, \& B. Verschuere (Eds.), Deception detection: New challenges and cognitive approaches (pp. 293-314). Chichester: Wiley Blackwell. https://doi.org/10.1002/9781118510001.ch13

Granhag, P. A., Rangmar, J., \& Strömwall, L. A. (2015). Small cells of suspects: Eliciting cues to deception by strategic interviewing. Journal of Investigative Psychology and Offender Profiling, 12, 127e141.

Grice, H. P. (1975). Logic and conversation. In P. Cole \& J. L. Morgan (Eds.), Syntax and semantics, Vol. 3, speech acts (pp. 41-58). New York, NY: Academic Press.

Jacobssen, R. R. (2010). The interpretation of indirect speech acts in relevance theory. Denmark: Kolding.

Kassin, S. M., \& McNall, K. (1991). Police interrogations and confessions: Communicating promises and threats by pragmatic implication. Law and Human Behavior, 15, 233-251. https://doi.org/10.1007/BF01061711

Kassin, S. M., Drizin, S. A., Grisso, T., Gudjonsson, G. H., Leo, R. A., \& Redlich, A. D. (2010). Police-induced confessions: Risk factors and recommendations. Law and Human Behavior, 34, 3-38. https://doi.org/10.1007/s10979-0099188-6

Luke, T. J., Dawson, E., Hartwig, M., \& Granhag, P. A. (2014). How awareness of evidence induces forthcoming counterinterrogation strategies. Applied Cognitive Psychology, 28, 876-882. https://doi.org/10.1002/acp.3019

Oleszkiewicz, S., Granhag, P. A., \& Kleinman, S. M. (2014). On eliciting intelligence from human sources: Contextualizing the Scharff-technique. Applied Cognitive Psychology, 28(6), 898-907. https://doi.org/10.1002/acp.3073

Sperber, D., \& Wilson, D. (1995). Relevance: Communication and Cognition (2nd ed.). Cambridge, MA: Oxford and Harvard University Press, Blackwell Publishing.

Srivatsav, M., Luke, T. J., Granhag, P.A., \& Vrij, A. (2019). Examining the effects of question framing on guilty suspects' inferences regarding interviewer's prior knowledge. Retrieved from osf.io/v485q

Tekin, S., Granhag, P. A., Strömwall, L. A., Mac Giolla, E., Vrij, A., \& Hartwig, M. (2015). Interviewing strategically to elicit admissions from guilty suspects. Law and Human Behavior, 39, 244-252. https://doi.org/10.1037/lhb0000131

Tekin, S., Granhag, P. A., Strömwall, L. A., \& Vrij, A. (2016). How to make perpetrators in denial disclose more information about their crimes. Psychology, Crime \& Law, 22, 561-580. https://doi.org/10.1080/1068316X.2016.1168425

How to cite this article: Srivatsav M, Luke TJ, Granhag PA, Vrij A. How do the questions asked affect suspects' perceptions of the interviewer's prior knowledge? J Investig Psychol Offender Profil. 2020;17: 160-172. https://doi.org/10.1002/jip.1546 